\title{
Neutrophil function and metabolism in individuals with diabetes mellitus
}

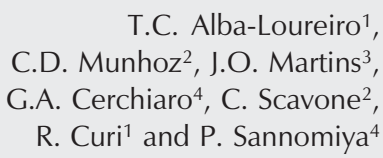

T.C. Alba-Loureiro',

C.D. Munhoz ${ }^{2}$, J.O. Martins ${ }^{3}$,

G.A. Cerchiaro ${ }^{4}$, C. Scavone ${ }^{2}$,

R. Curi ${ }^{1}$ and P. Sannomiya ${ }^{4}$

\author{
${ }^{1}$ Departamento de Fisiologia e Biofísica, ${ }^{2}$ Departamento de Farmacologia, \\ ${ }^{3}$ Departamento de Imunologia, Instituto de Ciências Biomédicas, \\ Universidade de São Paulo, São Paulo, SP, Brasil \\ ${ }^{4}$ Divisão de Experimentação, Instituto do Coração, LIM-11, \\ Faculdade de Medicina, Universidade de São Paulo, São Paulo, SP, Brasil
}

\begin{abstract}
Correspondence

P. Sannomiya

Divisão de Experimentação

InCor, LIM-11

Av. Dr. Enéas C. Aguiar, 44

05403-000 São Paulo, SP

Brasil

Fax: +55-11-3061-7178

E-mail: exppaulina@incor.usp.br

This study is dedicated to

the memory of Professor

João Garcia-Leme.

Research supported by FAPESP.

R. Curi and C. Scavone are

recipients of CNPq research

fellowships.

$\ldots \ldots \ldots \ldots \ldots \ldots \ldots$

Received March 9, 2007

Accepted May 21, 2007

Neutrophils act as first-line-of-defense cells and the reduction of their functional activity contributes to the high susceptibility to and severity of infections in diabetes mellitus. Clinical investigations in diabetic patients and experimental studies in diabetic rats and mice clearly demonstrated consistent defects of neutrophil chemotactic, phagocytic and microbicidal activities. Other alterations that have been reported to occur during inflammation in diabetes mellitus include: decreased microvascular responses to inflammatory mediators such as histamine and bradykinin, reduced protein leakage and edema formation, reduced mast cell degranulation, impairment of neutrophil adhesion to the endothelium and migration to the site of inflammation, production of reactive oxygen species and reduced release of cytokines and prostaglandin by neutrophils, increased leukocyte apoptosis, and reduction in lymph node retention capacity. Since neutrophil function requires energy, metabolic changes (i.e., glycolytic and glutaminolytic pathways) may be involved in the reduction of neutrophil function observed in diabetic states. Metabolic routes by which hyperglycemia is linked to neutrophil dysfunction include the advanced protein glycosylation reaction, the polyol pathway, oxygenfree radical formation, the nitric oxide-cyclic guanosine-3'-5'monophosphate pathway, and the glycolytic and glutaminolytic pathways. Lowering of blood glucose levels by insulin treatment of diabetic patients or experimental animals has been reported to have significant correlation with improvement of neutrophil functional activity. Therefore, changes might be primarily linked to a continuing insulin deficiency or to secondary hyperglycemia occurring in the diabetic individual. Accordingly, effective control with insulin treatment is likely to be relevant during infection in diabetic patients.
\end{abstract}

Key words

- Neutrophils

- Inflammation

- Diabetes mellitus

- Insulin

- Metabolism and cellular

function

\section{Diabetes mellitus and inflammation}

There is evidence that hormones are involved in the development of the inflammatory response. Inflammation evokes remark- able changes in the behavior of microvessels, hematogenous cells, and other reacting components. Though these changes are mainly brought about by release or activation of endogenous mediators, hormones play 
an important part in the control of inflammation $(1,2)$.

Several aspects have been shown to be impaired during inflammation in diabetes mellitus. These include decreased microvascular responses to inflammatory mediators such as histamine and bradykinin $(3,4)$, decreased protein leakage and edema formation (5-7), reduced mast cell degranulation (8), decreased leukocyte-endothelial cell interactions and reduced number of leukocytes in inflammatory lesions (9-15), lowered airway inflammatory response to antigen challenge $(16,17)$, low superoxide generation, and reduced release of tumor necrosis factor- $\alpha$ (TNF- $\alpha$ ), interleukin- $1 \beta$ and prostaglandin $\mathrm{E}_{2}$ by leukocytes upon exposure to lipopolysaccharide (LPS) (18-20); low content of arachidonic acid in neutrophils (20), and a reduction in lymph node retention capacity (21). These abnormalities might contribute to the increased susceptibility and severity of infections in diabetic patients.

In a series of experiments conducted in the early 1970's, Garcia-Leme and co-workers $(5,6)$ clearly demonstrated that insulin exerts direct regulatory effects on several stages of inflammation. Rats rendered diabetic by the administration of alloxan or by subtotal pancreatectomy fail to present the edema that follows the injection of chemical irritants or application of physical stimuli. This inhibition is abolished by previous injection of insulin and is not associated with increased blood glucose concentrations. In addition, diabetic rats exhibit decreased responses to permeability factors such as histamine, bradykinin, or serotonin injected into the skin compared to normal rats $(5,6)$. Light and electron microscopy studies have revealed that microvessels of alloxan diabetic rats challenged with histamine or serotonin exhibit less labeling by intravenously injected colloidal carbon particles than do vessels of normal animals (7). The integrity of the microcirculatory responses to noxious stimuli may consequently depend on the availability of insulin.

\section{Neutrophil function in diabetes mellitus}

Neutrophils play an essential role in the host inflammatory response against infection. Mowat and Baum (22) showed for the first time that the chemotactic activity of neutrophils from diabetic patients is significantly lower than in cells from healthy controls. Studies of the phagocytic and microbicidal activities of diabetic patients reveal, with few exceptions, an impairment of these functions. Decreased bactericidal activity (23), impairment of phagocytosis and decreased release of lysosomal enzymes (24), and reduced production of reactive oxygen species (25) by neutrophils of diabetic patients have been described. Furthermore, reduction in leukocyte phagocytosis and bactericidal activity showed a significant correlation with increases in blood glucose levels (26). It should be emphasized that investigation of well-controlled diabetic patients may fail to demonstrate any consistent defect that might predispose the patient to infection. However, in poorly controlled diabetic patients abnormalities in granulocyte chemotaxis, phagocytosis and microbicidal activity have been described by several groups.

Studies with diabetic rats and mice also showed a decreased neutrophil migration (912), phagocytosis capacity (27) and hydrogen peroxide production (28). Furthermore, the reduction of blood glucose levels by insulin treatment of diabetic patients (26) or rats (28) has been reported to be significantly correlated with improvement of neutrophil phagocytosis capacity.

During an inflammatory response, leukocytes roll along the lining endothelium of post-capillary venules and eventually become firmly attached to the vascular wall before migrating into tissues. Specific adhesion glycoproteins expressed on the surface 
of leukocytes and endothelial cells play a relevant role in the accumulation of leukocytes in the inflammatory lesion $(29,30)$. Members of the selectin family of cell adhesion molecules are thought to mediate leukocyte rolling along the walls of the microvasculature (29). Glycoproteins of the CD11/CD18 complex (B2integrins) expressed on leukocytes interact with ligands such as intercellular adhesion molecule-1 (ICAM-1) on endothelial cells to mediate leukocyte adhesion and migration (30). Monoclonal antibodies against cell adhesion molecules either on leukocytes or endothelial cells, or both can effectively inhibit inflammation (31). Accordingly, one possible explanation for the abnormal leukocyte function in diabetes mellitus might be a down-regulation of adhesion molecules that regulate leukocyte recruitment during the course of inflammatory processes.

The impaired local exudative cellular reaction in alloxan-induced diabetic rats is a consequence of defective leukocyte-endothelial interactions $(11,12,15)$. Intravital microscopic examination of the internal spermatic fascia microcirculatory network showed that a reduced number of leukocytes rolling along the venular endothelium is observed from the early stages of diabetes. Under the influence of an inflammatory stimulus, only few leukocytes accumulate in the perivascular tissue of diabetic rats in contrast to the massive number of leukocytes that normally emerge into the tissue around a vessel (12). These abnormalities are not related to changes in mean arterial pressure and heart rate values, blood flow velocity, venular shear rate, or blood glucose levels (15). Treatment of diabetic animals with insulin completely reverses defective leukocyte-endothelial interactions. Quantitation of the immune staining for ICAM-1 in postcapillary venules showed that, relative to naive rats, there is a significant increase in ICAM-1 protein levels in control rats under the influence of recombinant rat
TNF- $\alpha$. A similar effect is observed in ICAM1 mRNA levels, suggesting that TNF- $\alpha$ induces transcription and synthesis of ICAM1 in the microcirculation. However, in alloxan-induced diabetic rats increased levels of ICAM-1 mRNA are not accompanied by a similar increase in ICAM-1 protein levels. Furthermore, treatment of diabetic rats with insulin restores the expression of ICAM-1 on microvascular endothelium to values attained in control rats, without changes in the levels of mRNA for ICAM-1. The suggestion is that up-regulation of ICAM-1 induced by recombinant rat TNF- $\alpha$ depends on the availability of insulin (15). On the other hand, in LPS-induced lung inflammation, despite no significant differences in lung ICAM-1 and E-selectin immune staining between LPS-instilled diabetic rats and LPS-instilled controls, treatment of diabetic rats with insulin potentiates the expression of both adhesion molecules (19), suggesting that upregulation of both might be associated with the circulating levels of this hormone. Therefore, the number of leukocytes migrating to the lungs remarkably increases after treatment of diabetic rats with insulin when compared to values observed in LPS controls $(19,20)$.

\section{High glucose levels and neutrophil function in diabetes mellitus}

Another important advance in understanding the pathogenesis of neutrophil dysfunction and inflammatory disorders in diabetes is the observation that glucose or its analogues interact with proteins or lipids. The end products of this non-enzymatically catalyzed reaction, termed advanced glycation (glycosylation or glycoxidation) end products (AGEs), have been linked to the development of long-term complications of diabetes (32). Early glycation and oxidation processes result in the formation of reversible Schiff bases, which undergo an intramolecular rearrangement to form the Amadori 
products like glycated hemoglobin $\left(\mathrm{HbA}_{1 \mathrm{c}}\right)$ that is elevated in diabetic patients. A small proportion of these products undergo further slow and irreversible chemical rearrangements to form AGEs, which accumulate in the vasculature under conditions that are accelerated during hyperglycemia and when protein turnover is delayed (32). By interacting with several different receptors on endothelial cells, smooth muscle cells and infiltrating mononuclear phagocytes, AGEs modify cell structure and function leading to microvascular and macrovascular complications of diabetes (33). Non-enzymatic glycation of proteins may also interfere with leukocyte function. Masuda et al. (34) demonstrated that glycosylated proteins separated from the serum of diabetic rats decrease the membrane fluidity of leukocytes from control animals and may affect leukocyte migration. Corroborating these observations, aminoguanidine, an inhibitor of AGE formation, was shown to prevent the decreased leukocyte rolling behavior, as well as the reduced adhesion and migration of leukocytes in response to noxious stimuli observed in alloxan-diabetic rats (11). Indeed, the presence of a functional receptor for AGEs at the mRNA and protein level in human neutrophils is linked to a rise in intracellular $\mathrm{Ca}^{2+}$ and to actin polymerization (35). In addition, these investigators demonstrated impaired chemotactic peptide formylMet-Leu-Phe-induced migration of neutrophils through endothelial cell monolayers, suggesting that sustained stimulation of neutrophils with AGEs might reduce their ability to respond to physiological chemotactic stimuli (35).

The polyol pathway is another metabolic route by which hyperglycemia is linked to leukocyte dysfunction. Under physiological conditions, glucose is converted to glucose6-phosphate by hexokinase. When in excess, because the hexokinase pathway is saturated, glucose is converted to sorbitol by aldose reductase, a rate-limiting enzyme of the polyol pathway. Sorbitol is then converted to fructose by sorbitol dehydrogenase, and to fructose-3-phosphate by the action of 3-phosphokinase (36). Abnormalities in neutrophil functions have been shown to be associated with the polyol pathway. The impaired killing of Escherichia coli in diabetic patients is improved by treatment with ponalrestat, an aldose reductase inhibitor (37). During the course of an inflammatory response, the reduced number of adhered and migrated leukocytes presented by diabetic rats is not observed when the animals are treated with tolrestat, an aldose reductase inhibitor (13). These observations demonstrate a positive association between polyol pathway activation and leukocyte dysfunction in experimental diabetes mellitus. The hypothesis is that the accelerated formation of sorbitol in diabetic animals may increase the intracellular osmolarity or decrease the availability of the enzyme cofactor NADPH, leading to a disturbance of endothelial cell functions that might alter leukocyte-endothelial cell interactions. In fact, inhibition of the polyol pathway corrects the defective leukocyte-endothelial interaction found in experimental diabetes and may have a similar effect in diabetic patients (13).

Besides increased polyol pathway flux, the major metabolic change caused by hyperglycemia is an increase in oxygen-free radical formation, decreased resistance to oxidative stress and advanced protein glycosylation. All of these factors can trigger the development of diabetic complications (32). Antioxidant treatment can also be particularly important regarding decreased leukocyte migration. In support for this hypothesis, Zanardo et al. (14) demonstrated that vitamin $\mathrm{C}$ and probucol, antioxidant agents, correct the reduced cell migration in alloxandiabetic rats. On the other hand, experiments carried out on neutrophils showed that both inducible nitric oxide (NO) synthase activity and protein expression are increased in rats 
rendered diabetic by alloxan injection. In addition, treatment of these animals with NPH insulin (2 IU/day, for 3 days) reduces both inducible NO synthase activity and expression to normal levels (38). Cyclic guanosine-3'-5'-monophosphate (cGMP) content was determined in these cells for the investigation of the immediate second messenger effector of NO. Ten days after alloxan injection, basal levels of cGMP were increased in neutrophils from diabetic rats compared to controls. cGMP levels were reduced after treatment of diabetic animals with NPH insulin for at least 3 days. This information suggests that insulin modulates cGMP levels in neutrophils by NO production and that an increase in the NO-cGMP pathway may contribute to the impaired leukocyte function in diabetes mellitus (Figure 1).

\section{Neutrophil metabolism and diabetes mellitus}

The maximal activities of several enzymes of neutrophil metabolism such as hexokinase (glycolytic pathway) and citrate synthase (Krebs cycle) have been investigated by our group $(39,40)$. Also, the rates of metabolite production and utilization have been measured in incubated neutrophils $(28,39,40)$. The ATP required for neutrophil functions (22) is mainly produced by the metabolization of glucose to lactate (41). In fact, only 2-3\% of glucose is oxidized through the Krebs cycle in neutrophils (42). The stored glycogen can be utilized by neutrophils to obtain energy (43) mainly during the phagocytosis process. When the intracellular level of glucose is re-established, the resynthesis of glycogen is then initiated (44).

In addition to glucose, however, neutrophils also utilize glutamine at high rates (39). Glutamine is the most abundant amino acid in plasma and skeletal muscle, being produced in muscle, lung and liver (45). Glutamine utilized by neutrophils is mainly converted to glutamate, aspartate, lactate, and $\mathrm{CO}_{2}(39,46)$. The partial oxidation of glucose to lactate and the glutaminolytic pathway are the main source of ATP and of the production of intermediate metabolites for the synthesis of macromolecules such as fatty acids and phospholipids (40).

Many studies have shown metabolic alterations in neutrophils from diabetic patients. High levels of glucose and ketone bodies seem to influence neutrophil function by the production of polyols (47). Esmann (48) and Munroe and Shipp (49) did not observe differences in glucose utilization by neutrophils from healthy and diabetic patients, whereas decreased utilization of glucose by neutrophils was verified by others (50,51). Munroe and Shipp (49) did not observe alterations, whereas Martin et al. (50) and Esmann (51) found decreased production of lactate by neutrophils from diabetic patients. In our study (28), there was no alteration in glucose consumption or oxidation by neutrophils from streptozotocin-induced diabetic rats, but decreased lactate production and increased phosphofructokinase (PFK) maximal activity were observed (Figure 2). Similar results were observed in mesenteric lymph nodes and thymus lymphocytes from diabetic rats $(52,53)$. The activity of PFK is stimulated by fructose 2,6-biphosphate (54) and inhibited by ATP at low fructose 6-phosphate content, but not at high fructose 6-phosphate concentration (55). Therefore, under these conditions, the content of intermediates of glycolysis, such

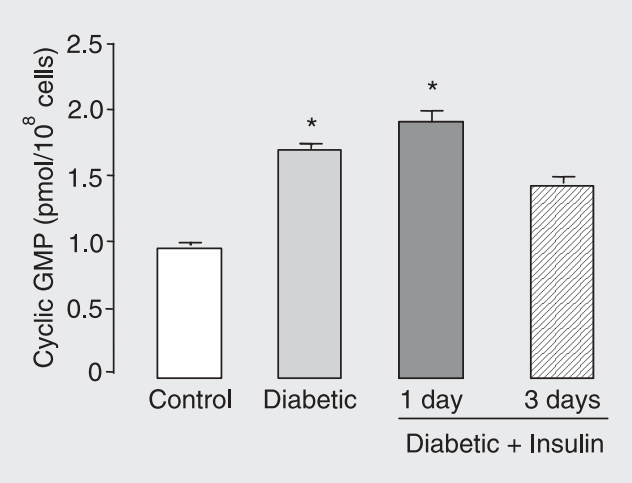

Figure 1. Cyclic GMP levels in neutrophils from diabetic rats, diabetic rats treated with insulin, and matching controls. Rats were rendered diabetic by the injection of alloxan $(42 \mathrm{mg} / \mathrm{kg}$, iv) 10 days before. Insulin (NPH, $2 \mathrm{IU} /$ day, sc) was given for 1 or 3 days before testing. Glycogenelicited peritoneal neutrophils from 3 to 4 rats were pooled, each animal yielding approximately $1 \times 10^{8}$ cells. Data are reported as pmol cyclic GMP per $10^{8}$ cells, as mean \pm SEM of 3 independent experiments (9 to 12 animals in each group). ${ }^{*} \mathrm{P}<$ 0.001 compared to control (ANOVA followed by the Newman-Keuls test). 
as fructose 6-phosphate and fructose 2,6biphosphate, is expected to be elevated in neutrophils. This may partially explain the increase in PFK activity. In agreement with this proposition, Moreno-Aurioles et al. (52) found increased fructose 2,6-biphosphate content and PFK activity in neutrophils from streptozotocin-induced diabetic rat.

Neutrophils from diabetic rats showed no significant change in glucose oxidation, citrate synthase, or $\mathrm{NAD}^{+}$- and $\mathrm{NADP}^{+}-$ linked isocitrate dehydrogenase activities, suggesting that the flux of substrates through the Krebs cycle was unchanged (28) (Figure 2 ). The pentose-phosphate pathway oxidizes glucose-6-phosphate to intermediates of the glycolytic pathway, generating NADPH and ribose-5-phosphate for fatty acid and nucleotide synthesis, respectively (56). NADPH is important for NADPH oxidase activity and for glutathione reductase to recycle oxidized glutathione in neutrophils (57).

Glutamine oxidation and glutaminase activity are significantly decreased in neutrophils from diabetic rats (28) (Figure 2). Glutamine plays an important role in protein (as an amino acid source), lipid (by NAD(P)H production) and nucleotide synthesis (by purine and pyrimidine production), and in NADPH oxidase activity (58). Glutamine raises the in vitro bacterial killing activity and the rate of reactive oxygen species production by neutrophils $(57,59)$. In addition, Pithon-Curi et al. (60) showed that glutamine has a protective effect on neutrophil apoptosis. Therefore, decreased glutamine utilization may contribute to the impaired inflammatory response in diabetic patients by increasing the occurrence of apoptosis in neutrophils.

These data, taken as a whole, show that neutrophils from diabetic rats present impaired metabolism of glucose and glutamine. On the other hand, palmitic acid oxidation is increased, and this may compensate for the reduction in glucose and glutamine utiliza-
Figure 2. Summary of the effects of diabetes mellitus on the metabolism of neutrophils. Red arrows indicate increase or decrease of enzymes and metabolite content of different pathways. Neutrophils from diabetic rats present impaired metabolism of glucose and glutamine. On the other hand, the increased fatty acid oxidation may compensate for the reduction in glucose and glutamine utilization to maintain the ATP supply for these cells. ACT-complex $=$ acyl carnitine transferase; $\mathrm{CS}=$ citrate synthase; FFA = free fatty acid; F-6$P=$ fructose-6-phosphate; $F-1,6$ $P=$ fructose-1, 6-biphosphate; $G$ 6-P = glucose-6-phosphate; HK $=$ hexokinase $; \mathrm{OOA}=$ oxaloace tic acid; PFK = phosphofructokinase.

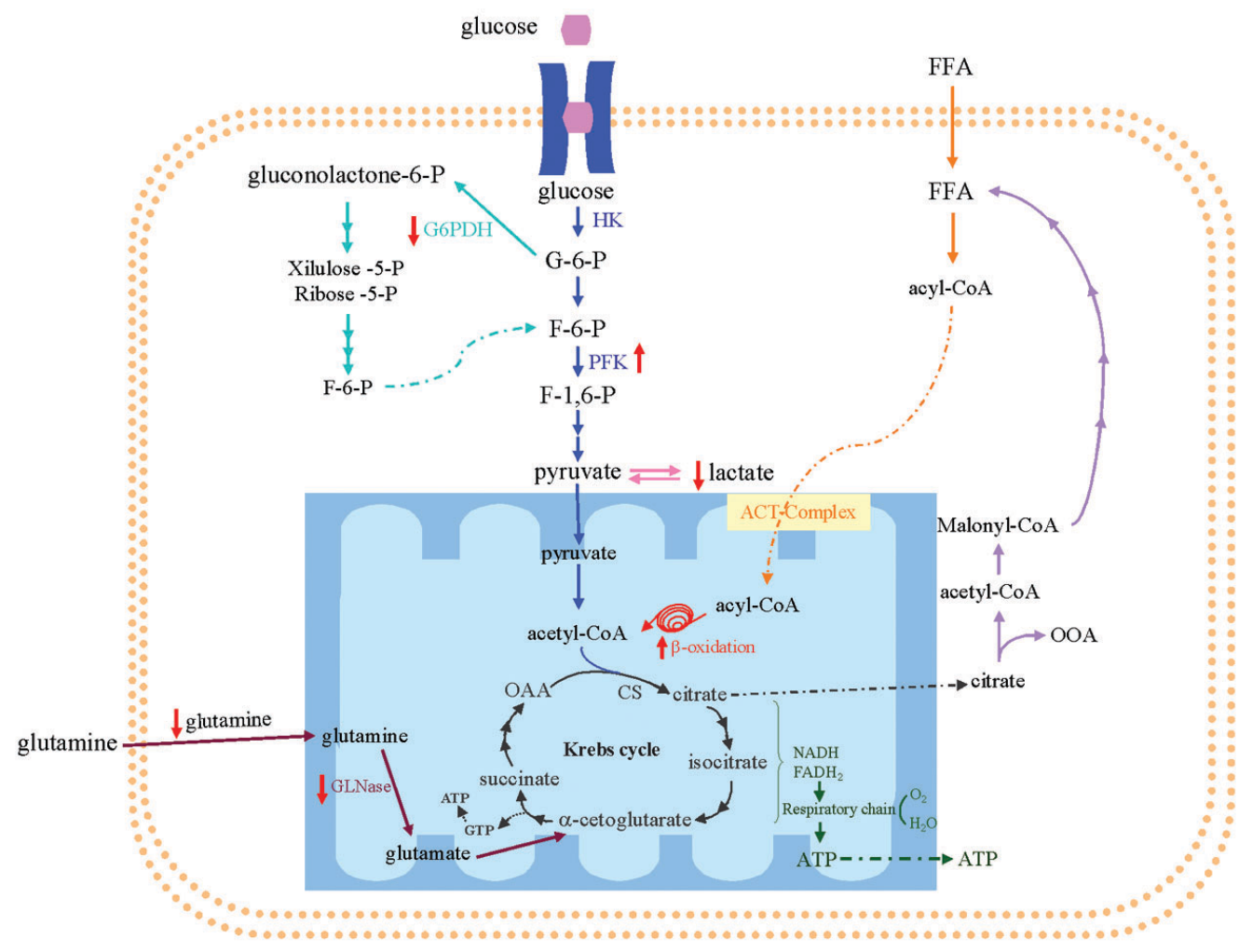


tion to maintain the ATP supply for these cells (28).

\section{Concluding remarks}

Survival depends on the ability of the host to respond appropriately to pathogenic challenges. It has long been recognized that many diabetic patients have a worse prognosis once infection is established. Abnormalities in neutrophil chemotaxis, phagocytosis and microbicidal mechanisms are responsible, at least in part, for the increased susceptibility to and severity of infections in diabetic patients. Considerable support to these clinical investigations was given by experimental studies on diabetic rats and mice. In addition, metabolic routes linked to leukocyte dysfunction, including advanced protein glycosylation, the polyol pathway, oxy- gen free radical formation, and the NO-cGMP pathway, have been described. Insulin restores the appropriate response to injury through a direct or indirect action on endothelial cells and leukocytes. For future studies the challenge remains to better understand the integration of both innate and adaptive immune systems with the endocrine and nervous systems, providing new insights into how inflammation is regulated.

\section{Acknowledgments}

We apologize to the many researchers whose work we have not been able to discuss in this limited review. The authors are indebted to E.P. Portiolli, G. de Souza, J.R. Mendonça, and L. de Sá Lima for constant assistance.

\section{References}

1. Garcia-Leme J. Hormones and inflammation. Boca Raton: CRC Press; 1989.

2. Garcia-Leme J, Farsky SP. Hormonal control of inflammatory responses. Mediat Inflamm 1993; 2: 181-198.

3. Fortes ZB, Garcia LJ, Scivoletto R. Vascular reactivity in diabetes mellitus: role of the endothelial cell. Br J Pharmacol 1983; 79: 771781.

4. Fortes ZB, Garcia LJ, Scivoletto R. Vascular reactivity in diabetes mellitus: possible role of insulin on the endothelial cell. Br J Pharmacol 1984; 83: 635-643.

5. Garcia LJ, Hamamura L, Leite MP, Rocha e Silva. Pharmacological analysis of the acute inflammatory process induced in the rat's paw by local injection of carrageenin and by heating. $\mathrm{Br} J$ Pharmacol 1973; 48: 88-96.

6. Garcia-Leme J, Bohm GM, Migliorini RH, de Souza MZ. Possible participation of insulin in the control of vascular permeability. Eur $J$ Pharmacol 1974; 29: 298-306.

7. Llorach MA, Bohm GM, Leme JG. Decreased vascular reactions to permeability factors in experimental diabetes. Br J Exp Pathol 1976; 57: 747-754.

8. Cavalher-Machado SC, de Lima WT, Damazo AS, de Frias Carvalho V, Martins MA, Silva PM, et al. Down-regulation of mast cell activation and airway reactivity in diabetic rats: role of insulin. Eur Respir $J$ 2004; 24: 552-558.

9. Pereira MA, Sannomiya P, Leme JG. Inhibition of leukocyte chemotaxis by factor in alloxan-induced diabetic rat plasma. Diabetes 1987; 36: 1307-1314.

10. Sannomiya P, Pereira MA, Garcia-Leme J. Inhibition of leukocyte chemotaxis by serum factor in diabetes mellitus: selective depres- sion of cell responses mediated by complement-derived chemoattractants. Agents Actions 1990; 30: 369-376.

11. Sannomiya P, Oliveira MA, Fortes ZB. Aminoguanidine and the prevention of leukocyte dysfunction in diabetes mellitus: a direct vital microscopic study. Br J Pharmacol 1997; 122: 894-898.

12. Fortes ZB, Farsky SP, Oliveira MA, Garcia-Leme J. Direct vital microscopic study of defective leukocyte-endothelial interaction in diabetes mellitus. Diabetes 1991; 40: 1267-1273.

13. Cruz JW, Oliveira MA, Hohman TC, Fortes ZB. Influence of tolrestat on the defective leukocyte-endothelial interaction in experimental diabetes. Eur J Pharmacol 2000; 391: 163-174.

14. Zanardo RC, Cruz JW, Martinez LL, de Oliveira MA, Fortes ZB. Probucol restores the defective leukocyte-endothelial interaction in experimental diabetes. Eur J Pharmacol 2003; 478: 211-219.

15. Anjos-Valotta EA, Martins JO, Oliveira MA, Casolari DA, Britto LR, Tostes RC, et al. Inhibition of tumor necrosis factor-alpha-induced intercellular adhesion molecule-1 expression in diabetic rats: role of insulin. Inflamm Res 2006; 55: 16-22.

16. Vianna EO, Garcia-Leme J. Allergen-induced airway inflammation in rats. Role of insulin. Am J Respir Crit Care Med 1995; 151: 809814.

17. Belmonte KE, Fryer AD, Costello RW. Role of insulin in antigeninduced airway eosinophilia and neuronal M2 muscarinic receptor dysfunction. J Appl Physiol 1998; 85: 1708-1718.

18. Boichot E, Sannomiya P, Escofier N, Germain N, Fortes ZB, Lagente V. Endotoxin-induced acute lung injury in rats. Role of insulin. Pulm Pharmacol Ther 1999; 12: 285-290.

19. de Oliveira MJ, Meyer-Pflug AR, Alba-Loureiro TC, Melbostad H, Costa da Cruz JW, Coimbra R, et al. Modulation of lipopolysaccha- 
ride-induced acute lung inflammation: Role of insulin. Shock 2006; 25: $260-266$.

20. Alba-Loureiro TC, Martins EF, Landgraf RG, Jancar S, Curi R, Sannomiya P. Role of insulin on PGE2 generation during LPSinduced lung inflammation in rats. Life Sci 2006; 78: 578-585.

21. Moriguchi P, Sannomiya P, Lara PF, Oliveira-Filho RM, Greco KV, Sudo-Hayashi LS. Lymphatic system changes in diabetes mellitus: role of insulin and hyperglycemia. Diabetes Metab Res Rev 2005; 21: 150-157.

22. Mowat A, Baum J. Chemotaxis of polymorphonuclear leukocytes from patients with diabetes mellitus. N Engl J Med 1971; 284: 621627.

23. Tan JS, Anderson JL, Watanakunakorn C, Phair JP. Neutrophil dysfunction in diabetes mellitus. J Lab Clin Med 1975; 85: 26-33.

24. Bagdade JD, Nielson KL, Bulger RJ. Reversible abnormalities in phagocytic function in poorly controlled diabetic patients. Am J Med Sci 1972; 263: 451-456.

25. Nielson CP, Hindson DA. Inhibition of polymorphonuclear leukocyte respiratory burst by elevated glucose concentrations in vitro. Diabetes 1989; 38: 1031-1035.

26. Jakelic J, Kokic S, Hozo I, Maras J, Fabijanic D. Nonspecific immunity in diabetes: hyperglycemia decreases phagocytic activity of leukocytes in diabetic patients. Med Arh 1995; 49: 9-12.

27. Panneerselvam S, Govindasamy S. Sodium molybdate improves the phagocytic function in alloxan-induced diabetic rats. Chem Biol Interact 2003; 145: 159-163.

28. Alba-Loureiro TC, Hirabara SM, Mendonca JR, Curi R, Pithon-Curi TC. Diabetes causes marked changes in function and metabolism of rat neutrophils. J Endocrinol 2006; 188: 295-303.

29. Rosen SD. Ligands for L-selectin: homing, inflammation, and beyond. Annu Rev Immunol 2004; 22: 129-156.

30. Mayadas TN, Cullere X. Neutrophil beta2 integrins: moderators of life or death decisions. Trends Immunol 2005; 26: 388-395.

31. Argenbright LW, Letts LG, Rothlein R. Monoclonal antibodies to the leukocyte membrane CD18 glycoprotein complex and to intercellular adhesion molecule-1 inhibit leukocyte-endothelial adhesion in rabbits. J Leukoc Biol 1991; 49: 253-257.

32. Brownlee M. Biochemistry and molecular cell biology of diabetic complications. Nature 2001; 414: 813-820.

33. Goldin A, Beckman JA, Schmidt AM, Creager MA. Advanced glycation end products: sparking the development of diabetic vascular injury. Circulation 2006; 114: 597-605.

34. Masuda M, Murakami T, Egawa H, Murata K. Decreased fluidity of polymorphonuclear leukocyte membrane in streptozocin-induced diabetic rats. Diabetes 1990; 39: 466-470.

35. Collison KS, Parhar RS, Saleh SS, Meyer BF, Kwaasi AA, Hammami $M M$, et al. RAGE-mediated neutrophil dysfunction is evoked by advanced glycation end products (AGEs). J Leukoc Biol 2002; 71: 433-444.

36. Hotta N. New approaches for treatment in diabetes: aldose reductase inhibitors. Biomed Pharmacother 1995; 49: 232-243.

37. Boland OM, Blackwell CC, Clarke BF, Ewing DJ. Effects of ponalrestat, an aldose reductase inhibitor, on neutrophil killing of Escherichia coli and autonomic function in patients with diabetes mellitus. Diabetes 1993; 42: 336-340.

38. Cerchiaro GA, Scavone C, Texeira S, Sannomiya P. Inducible nitric oxide synthase in rat neutrophils: role of insulin. Biochem Pharmacol 2001; 62: 357-362.

39. Curi TC, De Melo MP, De Azevedo RB, Zorn TM, Curi R. Glutamine utilization by rat neutrophils: presence of phosphate-dependent glutaminase. Am J Physiol 1997; 273: C1124-C1129.
40. Curi R, Newsholme P, Pithon-Curi TC, Pires-de-Melo M, Garcia C, Homem-de-Bittencourt Junior PI, et al. Metabolic fate of glutamine in lymphocytes, macrophages and neutrophils. Braz $J$ Med Biol Res 1999; 32: 15-21.

41. Beck WS, Valentine WN. The aerobic carbohydrate metabolism of leukocytes in health and leukemia. I. Glycolysis and respiration. Cancer Res 1952; 12: 818-822.

42. Beck WS. Occurrence and control of the phosphogluconate oxidation pathway in normal and leukemic leukocytes. J Biol Chem 1958; 232: 271-283.

43. Stjernholm RL, Burns CP, Hohnadel JH. Carbohydrate metabolism by leukocytes. Enzyme 1972; 13: 7-31.

44. Scott RB. Glycogen in human peripheral blood leukocytes. I. Characteristics of the synthesis and turnover of glycogen in vitro. $J$ Clin Invest 1968; 47: 344-352.

45. Curi R, Newsholme EA. The effect of adenine nucleotides on the rate and fate of glutamine utilization by incubated mitochondria isolated from rat mesenteric lymph nodes. Mol Cell Biochem 1989; 86: 71-76.

46. Ardawi MS, Newsholme EA. Glutamine metabolism in lymphocytes of the rat. Biochem J 1983; 212: 835-842.

47. Wilson RM, Tomlinson DR, Reeves WG. Neutrophil sorbitol production impairs oxidative killing in diabetes. Diabet Med 1987; 4: 37-40.

48. Esmann V. The diabetic leukocyte. Enzyme 1972; 13: 32-55.

49. Munroe JF, Shipp JC. Glucose metabolism in leucocytes from patients with diabetes mellitus, with and without hypercholesteremia. Diabetes 1965; 14: 584-590.

50. Martin SP, Chaudhuri SN, Green R, McKinney GR. The effect of adrenal steroids on aerobic lactic acid formation in human leukocytes. J Clin Invest 1954; 33: 358-360.

51. Esmann V. The polymorphonuclear leukocyte in diabetes mellitus. $J$ Clin Chem Clin Biochem 1983; 21: 561-567.

52. Moreno-Aurioles VR, Montano R, Conde M, Bustos R, Sobrino F. Streptozotocin-induced diabetes increases fructose 2,6-biphosphate levels and glucose metabolism in thymus lymphocytes. Life Sci 1996; 58: 477-484.

53. Otton R, Mendonca JR, Curi R. Diabetes causes marked changes in lymphocyte metabolism. J Endocrinol 2002; 174: 55-61.

54. Wegener G, Krause U. Different modes of activating phosphofructokinase, a key regulatory enzyme of glycolysis, in working vertebrate muscle. Biochem Soc Trans 2002; 30: 264-270.

55. Mansour TE. Studies on heart phosphofructokinase: purification, inhibition and activation. J Biol Chem 1963; 238: 2285-2292.

56. Casazza JP, Veech RL. The interdependence of glycolytic and pentose cycle intermediates in ad libitum fed rats. J Biol Chem 1986; 261: $690-698$.

57. Curi TC, De Melo MP, Palanch AC, Miyasaka CK, Curi R. Percentage of phagocytosis, production of $\mathrm{O}_{2}^{--}, \mathrm{H}_{2} \mathrm{O}_{2}$ and $\mathrm{NO}$, and antioxidant enzyme activities of rat neutrophils in culture. Cell Biochem Funct 1998; 16: 43-49.

58. Newsholme P, Lima MM, Procopio J, Pithon-Curi TC, Doi SQ, Bazotte RB, et al. Glutamine and glutamate as vital metabolites. Braz J Med Biol Res 2003; 36: 153-163.

59. Ogle CK, Ogle JD, Mao JX, Simon J, Noel JG, Li BG, et al. Effect of glutamine on phagocytosis and bacterial killing by normal and pediatric burn patient neutrophils. JPEN J Parenter Enteral Nutr 1994; 18: $128-133$.

60. Pithon-Curi TC, Schumacher RI, Freitas JJ, Lagranha C, Newsholme $\mathrm{P}$, Palanch AC, et al. Glutamine delays spontaneous apoptosis in neutrophils. Am J Physiol Cell Physiol 2003; 284: C1355-C1361. 